\title{
A CHARACTERIZATION OF SPECTRAL OPERATORS ON HILBERT SPACES
}

\author{
KOTARÓ TANAHASHI AND TAKASHI YOSHINO
}

\begin{abstract}
In [8] Wadhwa shows that if a bounded linear operator $T$ on a complex Hilbert space $H$ is a decomposable operator and has the condition (I), then $T$ is a spectral operator with a normal scalar part. In this paper, by using this result, we show that a weak decomposable operator $T$ is a spectral operator with a normal scalar part if and only if $T$ satisfies the assertion that (1) $T$ has the conditions (C) and (I) or that (2) every spectral maximal space of $T$ reduces $T$. This result improves [1, 6 and 7]. From this result, we can get a characterization of spectral operators, but this result does not hold in complex Banach space (see Remark 2).
\end{abstract}

1. Preliminaries. Let $H$ be a complex Hilbert space and $B(H)$ be the algebra of all bounded linear operators on $H$. Let $\sigma(T)$ be the spectrum of $T$ and $\operatorname{Lat}(T)$ be the family of all invariant subspaces of $T . Y \in \operatorname{Lat}(T)$ is called a spectral maximal space of $T$ if $Y$ contains all $Z \in \operatorname{Lat}(T)$ such that $\sigma(T \mid Z) \subset \sigma(T \mid Y)$. Let $\operatorname{SM}(T)$ be the family of all spectral maximal spaces of $T . T \in B(H)$ is called a decomposable (resp., weak decomposable) operator if for every finite open covering $\left\{G_{1}, \ldots, G_{n}\right\}$ of $\sigma(T)$, there exists a system $\left\{Y_{1}, \ldots, Y_{n}\right\}$ in $\operatorname{SM}(T)$ such that (1) $H=Y_{1}+\cdots+Y_{n}$ (resp., $H=\bar{Y}_{1}+\cdots+Y_{n}$ where $\bar{Y}$ denotes the closure of $\left.Y \subset H\right)$ and (2) $\sigma\left(T \mid Y_{i}\right) \subset$ $G_{i}$ for $i=1, \ldots, n . T \in B(H)$ is said to have the single valued extension property or the condition (A) if there exists no nonzero analytic function $f$ such that $(z-T) f(z) \equiv 0$. If $T \in B(H)$ has the condition (A), then for every $x \in H$, there exists a maximal open set $\rho_{T}(x)$ in the complex plane $C$ for which there exists the unique analytic function $x(z)$ such that $(z-T) x(z) \equiv x$ on $\rho_{T}(x)$. Let $\sigma_{T}(x)=$ $\rho_{T}(x)^{c}$ and $H_{T}(E)=\left\{x \in H \mid \sigma_{T}(x) \subset E\right\}$ for a subset $E$ of C. $T \in B(H)$ with (A) is said to have the condition (B) if there exists $K>0$ such that $\|x\| \leqslant K\|x+y\|$ for all $x$ and $y$ in $H$ with $\sigma_{T}(x) \cap \sigma_{T}(y)=\varnothing . T$ with (A) is said to have the condition (C) if $H_{T}(F)$ is closed for all closed sets $F$ in C. $T \in B(H)$ with (A) and (C) is said to have the condition (I) if $\sigma_{T}\left(P_{F} x\right) \subset \sigma_{T}(x)$ for all closed sets $F$ in $\mathrm{C}$ and for all $x \in H$ where $P_{F}$ is the orthogonal projection of $H$ onto $H_{T}(F)$.

It is known that weak decomposable operators have the condition (A) and that decomposable operators have the conditions (A) and (C) (see [2 and 5]). And if $T \in B(H)$ has the conditions (A) and (C), then $H_{T}(F) \in \operatorname{SM}(T)$ and $\sigma\left(T \mid H_{T}(F)\right)$ $\subset F$ for all closed sets $F$ in $\mathbf{C}$ (see [2]).

Received by the editors May 9, 1983.

1980 Mathematics Subject Classification. Primary 47B20, 47B40.

Key words and phrases. Spectral operator, decomposable operator, weak decomposable operator. 


\section{Main results.}

Lemma 1. If $T \in B(H)$ is a weak decomposable operator and if every spectral maximal space of $T$ reduces $T$, then $T$ has the condition $(\mathrm{C})$.

Proof. Let $F$ be a closed set in $\mathbf{C}$, and $G$ be any open set containing $F$. Since $\left\{F^{c}, G\right\}$ is an open covering of $\sigma(T)$, there exist $Y_{1}$ and $Y_{2}$ in $\operatorname{SM}(T)$ such that $H$ $=\overline{Y_{1}+Y_{2}}, \sigma\left(T \mid Y_{1}\right) \subset F^{c}$ and $\sigma\left(T \mid Y_{2}\right) \subset G$. If $x \in H_{T}(F)$, there exist $x_{n}^{i} \in Y_{i}$ for $i=1,2$ such that $x=\lim _{n \rightarrow \infty}\left(x_{n}^{1}+x_{n}^{2}\right)$. Let $P$ be the orthogonal projection of $H$ onto $Y_{1}$. Then we have $P T=T P$, hence $\sigma_{T}(P x) \subset \sigma_{T}(x) \cap \sigma\left(T \mid Y_{1}\right) \subset F \cap F^{c}=\varnothing$. This implies $P x=0$. Hence $0=P x=\lim _{n \rightarrow \infty}\left(P x_{n}^{1}+P x_{n}^{2}\right)=\lim _{n \rightarrow \infty}\left(x_{n}^{1}+P x_{n}^{2}\right)$, and so

$$
x=\lim _{n \rightarrow \infty}\left(x_{n}^{1}+x_{n}^{2}\right)-\lim _{n \rightarrow \infty}\left(x_{n}^{1}+P x_{n}^{2}\right)=\lim _{n \rightarrow \infty}\left(x_{n}^{2}-P x_{n}^{2}\right) .
$$

Since spectral maximal spaces are hyperinvariant (see [2, Proposition 1.3.2]), we have $x \in Y_{2}$. Hence $H_{T}(F) \subset Y_{2} \subset H_{T}\left(\sigma\left(T \mid Y_{2}\right)\right) \subset H_{T}(G)$. Since $G$ is any open set containing $F$, we have $H_{T}(F) \subset \cap Y_{2} \subset \cap H_{T}(G)=H_{T}(\cap G)=H_{T}(F)$. Thus $H_{T}(F)=\cap Y_{2}$ is closed.

LEMma 2. Let $T \in B(H)$ be a weak decomposable operator with $(\mathrm{B})$ and let $\overline{H_{T}(F)}$ reduce $T$ for all closed sets $F$ in $\mathrm{C}$. Then $T$ has the condition (C).

Proof. Let $F$ be a closed set in $\mathbf{C}$, and $G$ any open set containing $F$. Since $\left\{F^{c}, G\right\}$ is an open covering of $\sigma(T)$, there exist $Y_{1}$ and $Y_{2}$ in $\operatorname{SM}(T)$ such that $H=\overline{Y_{1}+Y_{2}}$, $\sigma\left(T \mid Y_{1}\right) \subset F^{c}$ and $\sigma\left(T \mid Y_{2}\right) \subset G$. If $x \in H_{T}(F)$, there exist $x_{n}^{i} \in Y_{i}$ for $i=1,2$ such that $x=\lim _{n \rightarrow \infty}\left(x_{n}^{1}+x_{n}^{2}\right)$. Let $P$ be the orthogonal projection of $H$ onto $\overline{H_{T}(F)}$. Then $P T=T P$ and $x=P x=\lim _{n \rightarrow \infty}\left(P x_{n}^{1}+P x_{n}^{2}\right)$. Since $Y_{1}$ is hyperinvariant, we have $P x_{n}^{1} \in Y_{1} \cap \overline{H_{T}(F)} \subset H_{T}\left(F^{c}\right) \cap \overline{H_{T}(F)}$. If $y \in H_{T}\left(F^{c}\right) \cap \overline{H_{T}(F)}$, then $y=$ $\lim _{n \rightarrow \infty} y_{n}$ where $y_{n} \in H_{T}(F)$ and $\sigma_{T}(y) \cap \sigma_{T}\left(-y_{n}\right) \subset F^{c} \cap F=\varnothing$. Hence $y=0$ because $\|y\| \leqslant K\left\|y-y_{n}\right\| \rightarrow 0(n \rightarrow 0)$ by the condition (B). Hence $P x_{n}^{1}=0$, and so $x=\lim _{n \rightarrow \infty} P x_{n}^{2} \in Y_{2}$ because $Y_{2}$ is hyperinvariant. Thus $H_{T}(F) \subset Y_{2} \subset H_{T}(G)$. The rest of the proof is similar to the proof of Lemma 1.

Lemma 3. Let $T \in B(H)$ be a weak decomposable operator. If $T$ has the conditions (C) and (I), then $T$ is a decomposable operator.

Proof. We show $H=H_{T}(F)+H_{T}\left(\overline{F^{c}}\right)$ for all closed sets $F$ in $\mathrm{C}$. Then it is easy to show that $T$ is a decomposable operator. Since $H=H_{T}(F) \oplus H_{T}(F)^{\perp}$, we have only to show $H_{T}(F)^{\perp} \subset H_{T}\left(\overline{F^{c}}\right)$. Let $G$ be any open set containing $F$. Since $\left\{F^{c}, G\right\}$ is an open covering of $\sigma(T)$, there exist $Y_{1}$ and $Y_{2}$ in $\operatorname{SM}(T)$ such that $H=\overline{Y_{1}+Y_{2}}$, $\sigma\left(T \mid Y_{1}\right) \subset F^{c}$ and $\sigma\left(T \mid Y_{2}\right) \subset G$. If $x \in H_{T}(\bar{G})^{\perp}$, there exist $x_{n}^{i} \in Y_{i}$ for $i=1,2$ such that $x=\lim _{n \rightarrow \infty}\left(x_{n}^{1}+x_{n}^{2}\right)$. Hence

$$
0=P_{\bar{G}} x=\lim _{n \rightarrow \infty}\left(P_{\bar{G}} x_{n}^{1}+P_{\bar{G}} x_{n}^{2}\right)=\lim _{n \rightarrow \infty}\left(P_{\bar{G}} x_{n}^{1}+x_{n}^{2}\right),
$$

and so

$$
x=\lim _{n \rightarrow \infty}\left(x_{n}^{1}+x_{n}^{2}\right)-\lim _{n \rightarrow \infty}\left(P_{\bar{G}} x_{n}^{1}+x_{n}^{2}\right)=\lim _{n \rightarrow \infty}\left(x_{n}^{1}-P_{\bar{G}} x_{n}^{1}\right) .
$$


Since $\sigma_{T}\left(P_{\bar{G}} x_{n}^{1}\right) \subset \sigma_{T}\left(x_{n}^{1}\right) \subset \sigma\left(T \mid Y_{1}\right) \subset F^{c}$, we have $x_{n}^{1}-P_{\bar{G}} x_{n}^{1} \in H_{T}\left(\overline{F^{c}}\right)$ and so $x \in H_{T}\left(\overline{F^{c}}\right)$. Hence $H_{T}(\bar{G})^{\perp} \subset H_{T}\left(\overline{F^{c}}\right)$, and so $H_{T}(\bar{G}) \supset H_{T}\left(\overline{F^{c}}\right)^{\perp}$. Since $G$ is any open set containing $F$, we have $H_{T}(F)=H_{T}(\cap \bar{G})=\cap H_{T}(\bar{G}) \supset H_{T}\left(\overline{F^{c}}\right)^{\perp}$. Thus $H_{T}(F)^{\perp} \subset H_{T}\left(\overline{F^{c}}\right)$.

THEOREM. If $T \in B(H)$, then the following assertions are equivalent.

(1) $T=N+Q$ where $N \in B(H)$ is a normal operator and $Q$ is a quasinilpotent operator commuting with $N$.

(2) $T$ is a weak decomposable operator with (C) and (I).

(3) $T$ is a weak decomposable operator and every spectral maximal space of $T$ reduces $T$.

(4) $T$ is a weak decomposable operator with (B), and $\overline{H_{T}(F)}$ reduces $T$ for all closed set $F$ in $\mathbf{C}$.

Proof. We show the implications (1) $\Rightarrow(4) \Rightarrow(2) \Rightarrow(1)$ and (1) $\Rightarrow(3) \Rightarrow(2)$.

$(1) \Rightarrow(4)$ and (3). This implication is known. But we include it for completeness. (1) implies that $T$ is a spectral operator with a normal scalar part $N$. Hence $T$ is a decomposable operator and $T$ has the conditions (B) and (C). Hence $T$ is a weak decomposable operator. Let $E()$ be the resolution of the identity of $N$. Then $H_{T}(F)=H_{N}(F)=E(F) H$ for all closed sets $F$ in $\mathrm{C}$. This implies $H_{T}(F)$ reduces $T$. And if $Y \in \operatorname{SM}(T)$, then $Y=H_{T}(\sigma(T \mid Y))$, hence $Y$ reduces $T$ (see, for details, [2 and 3]).

(4) $\Rightarrow(2)$. $T$ has the condition (C) by Lemma 2. Since $P_{F} T=T P_{F}$ for all closed sets $F$ in $\mathbf{C}, T$ has the condition (I).

(2) $\Rightarrow(1)$. Since $T$ is a decomposable operator with (I) by Lemma 3, this implication follows from [8].

(3) $\Rightarrow$ (2). $T$ has the condition (C) by Lemma 1 , hence $H_{T}(F) \in \operatorname{SM}(T)$ for all closed sets $F$ in C. Thus $P_{F} T=T P_{F}$ and $T$ has the condition (I).

Since $T \in B(H)$ is a spectral operator if and only if $T$ is similar to some $S \in B(H)$ which satisfies the condition (1) of the Theorem (see [3]), we have the following

Corollary. $T \in B(H)$ is a spectral operator if and only if $T$ is similar to some $S \in B(H)$ which satisfies one of the conditions of the Theorem.

REMARK 1. The condition (I) is introduced by Wadhwa in [8]. In [6] Jafarian proved the implication $(2) \Rightarrow(1)$ of the Theorem under an assumption that $T$ is reductive, i.e. every invariant subspace of $T$ reduces $T$.

REMARK 2. Let $X=L^{\infty}[0,1]$ be the Banach space of all essentially bounded complex valued functions on $[0,1]$ endowed with the essential supremum norm. Let $T \in B(X)$ be the multiplication operator, i.e. $(T x)(t)=t x(t)$ for $t \in[0,1]$ and $x \in X$. Then we can show that $T$ is a decomposable operator and $\sigma_{T}(x)=\operatorname{ess} \operatorname{supp} x$ by an argument similar to [4, p. 106]. $X_{T}(F)$ stands for $H_{T}(F)$. Define $P_{F} \in B(X)$ such that $\left(P_{F} x\right)(t)=\chi_{F}(t) x(t)$ for $t \in[0,1]$ where $\chi_{F}(t)$ is the characteristic function of a closed set $F$ in $\mathbf{C}$. Then $P_{F}$ is a projection of $X$ onto $X_{T}(F)$ (of course, $P_{F}$ is not selfadjoint) and $P_{F} T=T P_{F}$. Hence $X_{T}(F)$ reduces $T$, and $T$ has the 
condition (I). And if $\sigma_{T}(x) \cap \sigma_{T}(y)=\varnothing$, then $|x(t)+y(t)|=|x(t)|+|y(t)|$ a.e. $[0,1]$. Hence $\|x+y\| \geqslant\|x\|$, thus $T$ has the condition (B). But $T$ is not a spectral operator. Because if $T$ is spectral, then $T$ has the resolution of the identity $E($ ). Hence, $x=\lim _{n \rightarrow \infty} x_{n}$ where $x_{n}=E(\{0\} \cup[1 / n, 1]) x$ for all $x \in X$. Let $x(t) \equiv 1$. Since $E(F) X=X_{T}(F)$ for all closed sets $F$ in C, ess supp $x_{n} \subset\{0\} \cup[1 / n, 1]$. Hence $\left\|x-x_{n}\right\|=1$ for all $n$. This is a contradiction. Thus, the Theorem does not hold in this case.

\section{REFERENCES}

1. E. Albrecht, A characterization of spectral operators on Hilbert spaces, Glasgow Math. J. 23 (1982), 91-95.

2. I. Colojoară and C. Foiaş, Theory of generalized spectral operators, Gordon \& Breach, New York, 1968.

3. N. Dunford and J. T. Schwartz, Linear operators. Part III: Spectral operators, Wiley, New York, 1971.

4. I. Erdelyi and R. Lange, Spectral decomposition on Banach spaces, Lecture Notes in Math., vol. 623. Springer-Verlag, New York, 1977.

5. A. A. Jafarian, Weak and quasidecomposable operators, Rev. Roumaine Math. Pures Appl. 22 (1977), 195-212.

6. __ On reductive operators, Indiana Univ. Math. J. 23 (1974), 607-613.

7. K. Tanahashi, Reductive weak decomposable operators are spectral, Proc. Amer. Math. Soc. 87 (1983), 44-46.

8. B. L. Wadhwa, Decomposable and spectral operators on a Hilbert space, Proc. Amer. Math. Soc. 40 (1973), 112-114.

9. __ A note un reductive operators, Acta Sci. Math. (Szeged) 38 (1976), 187-189.

TOhoku College of Pharmacy, Komatsushima, Sendai, 983, Japan

Department of Mathematics, College of General Education, TOHoku University, Kawauchi, SENDAI, 980, JAPAN 